\title{
Descarte de Medicamentos Residencial: Uma Revisão Integrativa
}

\author{
Cleidivania Glécia Pereira, ${ }^{1}$ Annalu Moreira Aguiar, ${ }^{2}$ \\ Rafael de Carvalho Mendes, ${ }^{3}$ Ana Emilia Formiga Marques ${ }^{4}$
}

\section{RESUMO}

A RDC 222/2018 determina como os estabelecimentos que prestam serviços de saúde devem elaborar um gerenciamento de resíduos, porém essa RDC não abrange o descarte feito pelo consumidor. O descarte residencial é um conteúdo que precisa ser mais abordado, pois o ato do descarte incorreto vem provocando grande impacto ao meio ambiente. O estudo tem como objetivo identificar como e onde os usuários realizam o descarte de medicamentos residencial. Este estudo trata-se de uma revisão integrativa, e foram usados os descritores: Descarte de medicamentos, impacto ambiental, recolhimentos de fármacos, conscientização dos usuários. Os critérios de inclusão foram: artigos em português, publicados no período de 2009 a 2019 e que abordasse o tema de forma direta; e os de exclusão foram: artigos duplicados nas bases de dados, artigos de revisão sistemática, artigos que não abordavam o tema específico do trabalho e que não contemplava o período de 2009 a 2019, como busca nas bases de dados BVS e SciELO. A busca resultou em 119 artigos na base de dados BVS e 21 na base de dados SciELO; em seguida foram aplicados os filtros com critérios de inclusão e realizada uma leitura prévia, resultando, assim, em dez artigos que abordavam o tema pesquisado de forma clara. A avaliação dos estudos evidencia a necessidade de criação de medidas para melhorar o quadro atual, pois a maioria da população não recebe orientações ou não tem opção para descartar medicamentos em desusos.

Palavras-chave: fármacos; coleta de resíduos sólidos; impacto ambiental.

\section{DISPOSAL OF RESIDENTIAL MEDICINES: AN INTEGRATIVE REVIEW}

\section{ABSTRACT}

Over time, various diseases have been suggested, so the pharmaceutical industry has found the need to produce new pharmaceutical products, consequently, there has been an increase in the disposal of these drugs. RDC 222/2018, determines how establishments that provide health services should develop waste management, however this RDC does not cover consumer disposal. Residential disposal is a content that needs to be addressed more, as the act of incorrect disposal has caused a great impact on the environment. The study aims to identify how and where users dispose of residential medicines. This study is an integrative review and aims to address the disposal of residential drugs. Using the descriptors: Disposal of medicines, environmental impact, drug withdrawals, user awareness. The inclusion criteria were articles in Portuguese, published in the period from 2009 to 2019 and that addressed the topic directly and the exclusion criteria were: duplicate articles in the databases, systematic review articles, articles that did not address the specific topic of the work, and which did not include the period from 2009 to 2019 as a search in the VHL and SciELO databases. The search resulted in 119 in the VHL database and 21 in the SciELO database, after which the filters with inclusion and exclusion criteria were applied, and a previous reading was carried out, thus resulting in 10 articles in which they addressed the researched topic clearly. The evaluation of the studies shows the need to implement measures to improve the current situation, since most of the population does not receive guidance or has no option to discard medicines that are no longer used.

Keywords: drugs; solid waste collection; environmental impact.

RECEBIDO EM: 9/7/2020

MODIFICAÇÕES SOLICITADAS EM: 27/1/2021

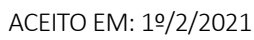

\footnotetext{
${ }^{1}$ Faculdade de Medicina Estácio de Juazeiro do Norte. Juazeiro do Norte/CE, Brasil.

2 Faculdade de Medicina Estácio de Juazeiro do Norte. Cajazeiras/PB, Brasil.

${ }^{3}$ Faculdade de Medicina Estácio de Juazeiro do Norte. Cajazeiras/PB, Brasil.

${ }^{4}$ Autora correspondente. Faculdade de Medicina Estácio de Juazeiro do Norte. Avenida Brasil, 393 - Jardim Adalgisa. Cajazeiras/PB, Brasil. CEP 58900-000. http://lattes.cnpq.br/5041426851854678. https://orcid.org/0000-0003-1568-7231. anaemiliaformiga@hotmail.com
} 


\section{INTRODUÇÃO}

A produção de medicamento em escala industrial foi um grande avanço para a saúde pública e uma inovação nas atividades terapêuticas. Em todos os países toneladas desses medicamentos são produzidos em alta demanda, com o objetivo de melhorar a qualidade de vida da população. Por influência da mídia ou de pessoas conhecidas e a facilidade de adquirir essas substâncias, ocorre um aumento no consumo de medicamentos pela população no Brasil (PINTO et al., 2015; TRIBESS JUNIOR; BELLAVER; ZANCANARO, 2018).

Consequentemente, surge mais uma questão para a saúde pública. O destino desses medicamentos residenciais. Na maioria das vezes terminam sendo descartados em lixo comum ou em esgotos pela população, e já foi possível identificar a presença de vários fármacos nos rios de vários países. Como resultado, ocorre uma contaminação tanto na água quanto no solo (LIMA et al., 2016; JORGE JOÃO, 2011).

A Resolução da Diretoria Colegiada - RDC no 222 de 2018 - dispõe sobre o regulamento técnico para o gerenciamento de resíduos de serviços de saúde. Essa lei define que os estabelecimentos que prestam serviços de saúde para a população devem instituir um gerenciamento dos resíduos gerados pelos serviços, cujo objetivo principal é a redução desses resíduos altamente tóxicos, visando à segurança para a saúde pública e o meio ambiente (BRASIL, 2018).

A resolução Conama no 358 de 2005 dispões sobre o tratamento e a disposição final dos resíduos dos serviços de saúde e dá outras providências. Define a necessidade de adotar providências para diminuir a produção de resíduos. Algumas dessas sugestões é a substituição de materiais com um menor risco de contaminação e incentivar a reciclagem de produtos. Incorporar medidas preventivas possui mais vantagens do que medidas corretivas, reduzindo com mais facilidade a contaminação do meio ambiente e o prejuízo provocado à saúde pública (BRASIL, 2005).

Essas leis mostram como os estabelecimentos que prestam serviços relacionados com os atendimentos à saúde humana ou animal, incluindo os serviços de laboratórios, farmácias e outros, devem gerenciar os resíduos sólidos que eles geraram. Essas leis que foram desenvolvidas para esses perfis de descarte, porém, não incluem o descarte feito pelo consumidor e não obrigam as farmácias e os outros estabelecimentos a recolherem esses produtos (UEDA et al., 2009).

A falta de um programa de recolhimento para medicamentos fora da data de validade ou que não vão ser mais utilizados, agrava mais ainda a situação, pois a quantidade de fármacos detectados constantemente no trato de esgoto, nas águas e no solo, em intensas concentrações, vem provocando uma grande preocupação. De acordo com estudos, alguns cânceres podem estar associados com a quantidade e o tempo em que esses fármacos ficam presentes no meio ambiente (BILA; DEZOTTI, 2003; SOUZA; FALQUETO, 2015).

No Brasil, o descarte inadequado de medicamentos ainda é um ato muito praticado por uma grande parte da população, na maioria das vezes por falta de informações ou pela falta de um programa de recolhimento desses medica-

Editora Unijuí - Revista Contexto \& Saúde - ISSN 2176-7114 - v. 21, n. 43, jul./set. 2021 
mentos. A conscientização da população para a realização do descarte correto de medicamentos é uma excelente medida a ser empregada, pois a melhor forma de evitar uma contaminação é impedir o contato dessas substâncias com o meio ambiente (BLANKENSTEIN; PHILIPPI JUNIOR, 2018).

O estudo, portanto, é de extrema importância para trazer maior conhecimento sobre como é realizado o descarte de medicamentos pela população, e poderá contribuir significativamente para a sensibilização sobre os prejuízos que a prática do descarte inadequado pode causar tanto para o meio ambiente quanto, também, para a saúde da população. Esta revisão integrativa objetiva identificar estudos que evidenciem como os usuários executam a prática de descarte de medicamentos.

\section{MÉTODO}

Esta investigação configura uma revisão integrativa, que busca estudos e informações sobre os descartes de medicamentos. A partir disso, serão avaliados e interpretados os resultados para apresentar uma revisão/síntese do conhecimento disponível em bases de dados.

Para essa revisão foi realizado um levantamento de dados nas plataformas virtuais de bases de dados Biblioteca Virtual em Saúde (BVS) e Biblioteca Eletrônica Científica On-line (SciELO), utilizando os descritores coleta de resíduos sólidos, impacto ambiental, recolhimentos de fármacos, conscientização dos usuários. Os estabelecimentos de critérios de inclusão foram: artigos em português que abordassem diretamente o tema no período de 2009 a 2019. Os critérios de exclusão foram: artigos duplicados, revisões integrativas, monografias, dissertações, teses, plataformas pagas.

\section{RESULTADOS E DISCUSSÕES}

$\mathrm{Na}$ base de dados BVS foram encontrados 119 artigos, e na base de dados SciELO 21; logo em seguida foi aplicado o filtro de critérios de inclusão, e obteve-se um resultados de 44 artigos na BVS e 10 na SciELO; logo após foi realizada uma leitura prévia que resultou na eliminação dos artigos que se encontravam repetidos nas bases de dados, incompletos e aqueles que não estavam diretamente relacionados com o tema. Foi efetuada uma leitura crítica, verificando quais os autores, temas, objetivos, resultados e conclusões de maior relevância.

A busca na literatura resultou em 21 artigos, e, desses, apenas 10 enquadravam-se e abordavam o tema sobre descarte de medicamentos com clareza, como demonstra, de forma simplificada, o fluxograma da Figura 1. 
Figura 1 - Fluxograma do processo de seleção dos artigos nos bancos de dados acessados
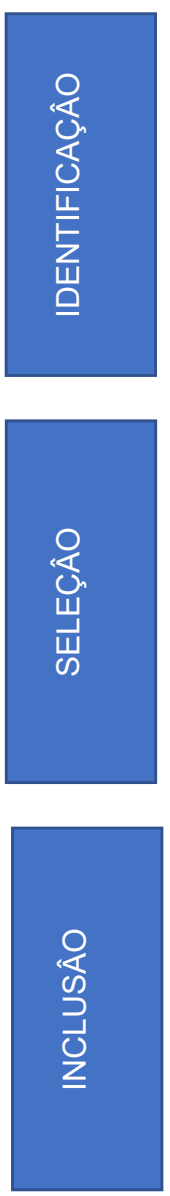
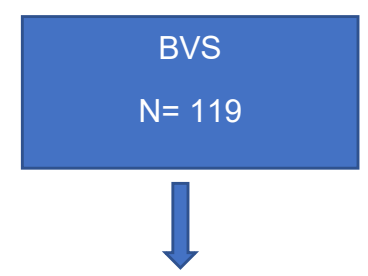

Artigos eliminados por não estarem no idioma português e não estarem no período de 2009 á 2019 $\mathrm{N}=86$

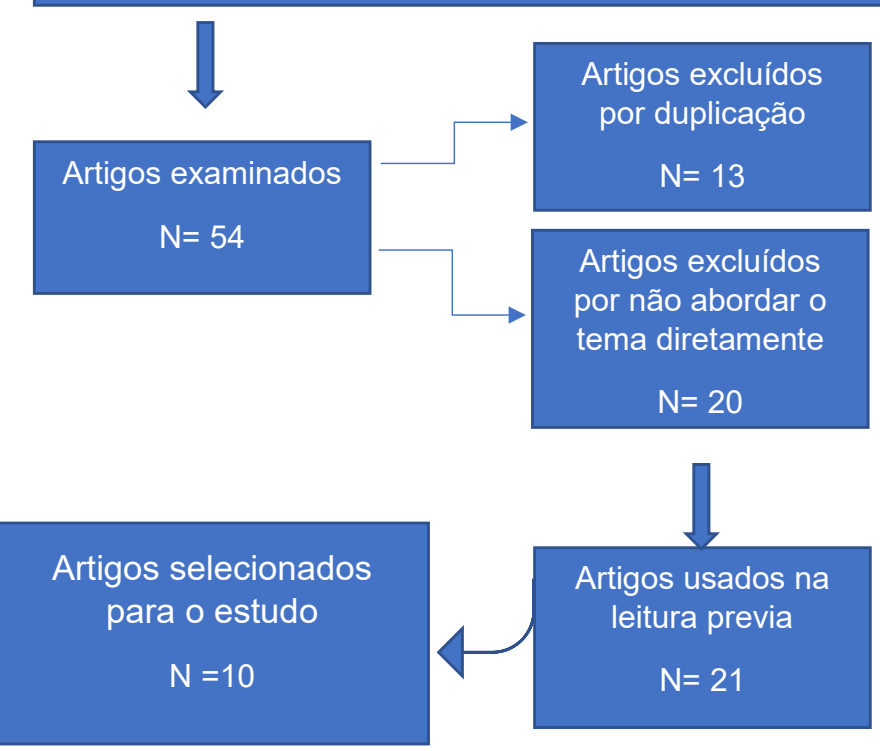

Fonte: Dados da pesquisa (2020). 


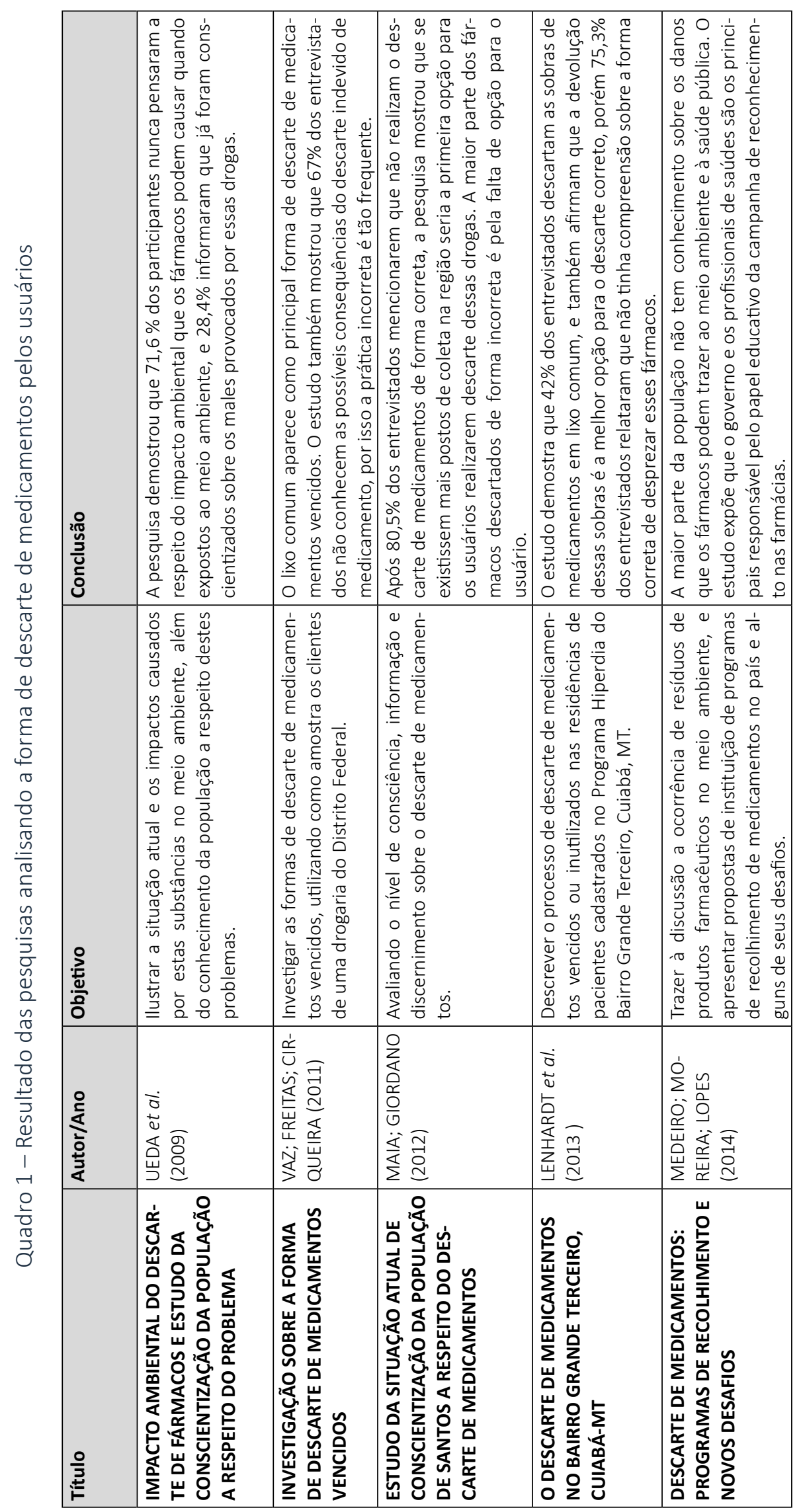




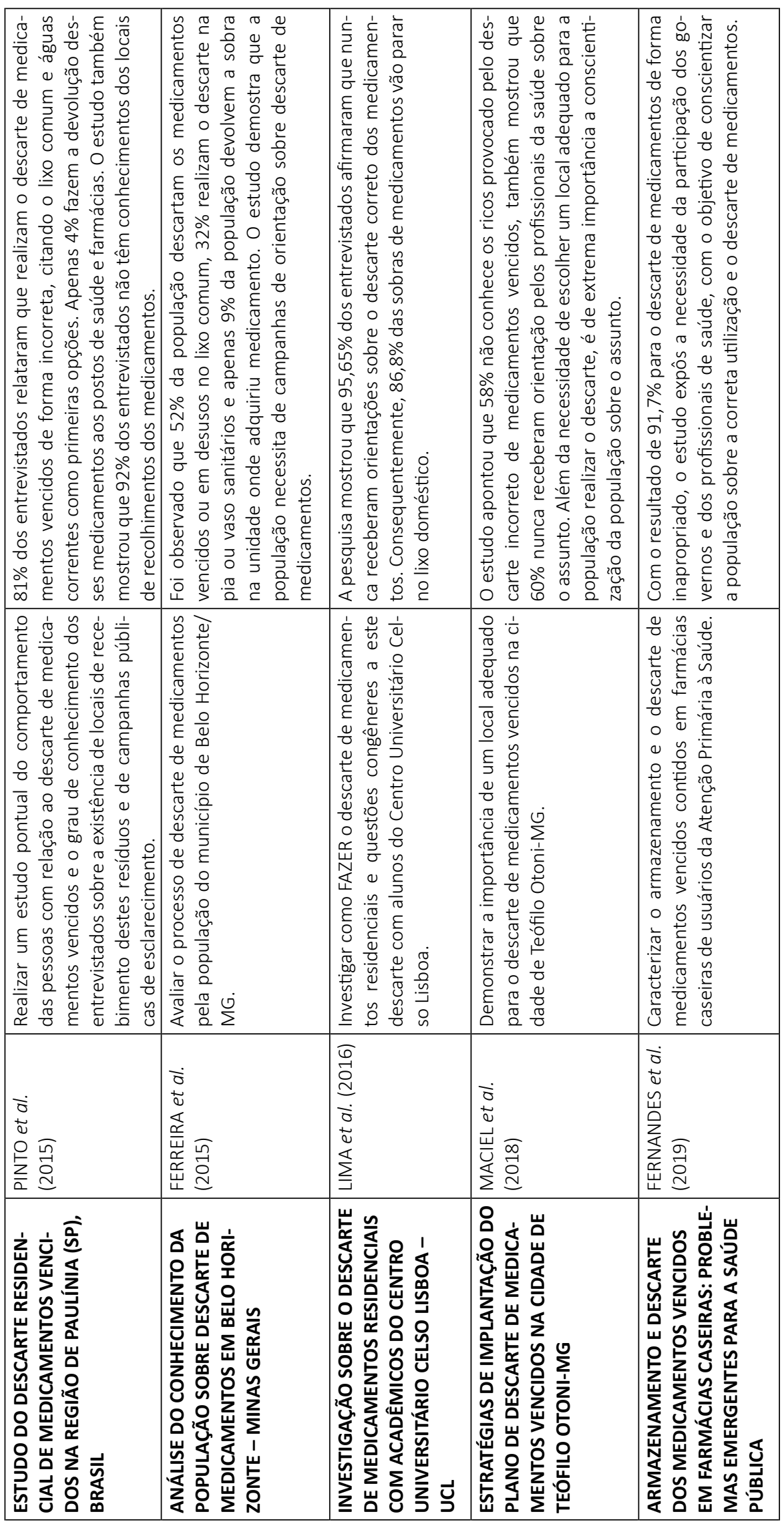


Na revisão integrativa realizada evidenciou-se que o descarte de medicamentos residencial é um tema que precisa ser bastante discutido. Lima et al. (2016), em seu estudo, afirmam que o descarte inadequado de medicamentos está ligado diretamente ao nível de conhecimento dos usuários, e a falta de informações obriga os usuários a descartar esses fármacos em lugares impróprios. A pesquisa realizada pelos mesmos mostrou que a maioria dos entrevistados não acredita que a prática do descarte incorreto pode causar algum dano ao meio ambiente.

Uma pesquisa de Ferreira et al. (2015) em Belo Horizonte - Minas Gerais - mostrou como primeira opção para descartar os medicamentos em desuso ou fora do prazo de validade o lixo comum, e em segundo lugar ficaram o vaso sanitário ou a pia. A ausência de conhecimento da população sobre esse assunto é preocupante, pois ainda não há um cuidado exclusivo para o descarte de medicamentos residencial, e esses resíduos sólidos acabam sendo destinados em lixões, consequentemente colocando em risco a saúde dos seres vivos e prejudicando o meio ambiente. Lenhardt et al. (2013), em seu estudo, afirmam que o descarte residencial é feito de forma indevida, a maioria sendo realizada em lixo doméstico. Segundo esses autores, os usuários devem receber orientação sobre a forma correta de descarte, e as unidades de saúde devem estar preparadas para receber as sobras de medicamentos da população.

Segundo Vaz, Freitas e Cirqueira (2011), o acúmulo de medicamentos em residências aumenta o risco de descarte inadequado; geralmente esse descarte irregular é realizado por falta de opção. Com isso Ueda et al. (2009) apontam a necessidade da instituição de pontos de coleta de medicamentos em locais estratégicos, além de investir mais em campanhas educacionais sobre a importância do descarte correto e dos danos gerados por esses fármacos ao solo. Maia e Giordano (2012) acreditam que se houvesse mais postos de coleta seria a primeira opção de descarte dos usuários.

Pinto et al. (2015) evidenciaram a falta de interesse por parte do poder público em investir em campanhas para a conscientização da população sobre a importância do descarte correto de medicamentos, visando à diminuição dos danos causados à saúde pública. Essa divulgação é de extrema importância, podendo ser utilizados os principais meios de comunicação, como TV, rádio, internet, etc., visando a um alcance maior para a conscientização, uma vez que a maioria dos cidadãos desconhece os danos que esses atos podem causar.

Lima et al. (2016) acreditam que a orientação para que os usuários realizem o descarte correto deve ser feita, principalmente, pelos profissionais de saúde. Esses profissionais devem orientar a população sobre os danos que esses fármacos podem trazer ao meio ambiente e à saúde pública, e afirmam, em seu estudo, que é fundamental o investimento em capacitações específicas sobre o assunto para os agente comunitários, pois esses profissionais já realizam um trabalho muito importante junto com a sociedade, podendo incluir a orientação sobre a forma correta do descarte de medicamentos.

Ferreira et al. (2015) e Medeiro, Moreira e Lopes (2014), em seus estudos, afirmam que os farmacêuticos são os últimos profissionais da saúde a ter contato direto com os pacientes, sendo a eles atribuído o dever de garantir a

Editora Unijuí - Revista Contexto \& Saúde - ISSN 2176-7114 - v. 21, n. 43, jul./set. 2021 
segurança e a eficácia, promovendo sempre o uso racional de medicamentos, visando a garantir proteção à sociedade. Esses profissionais devem ser uma fonte de informações para a população. Além da orientação sobre o uso correto dos medicamentos, os farmacêuticos devem informar aos pacientes o local mais adequado para o mesmo realizar o descarte desses fármacos. Segundo Maciel et al. (2018), a falta de comunicação entre o farmacêutico e os pacientes sobre o assunto pode induzir a população a realizam o descarte em lixo comum.

\section{CONCLUSÃO}

No estudo realizado foi possível identificar que o lixo comum e o esgoto são as opções mais utilizadas para desprezar esses fármacos que não serão mais utilizados. Esse descarte incorreto ocorre pela falta de opção e de orientação, posto que uma grande parte da população não tem conhecimento sobre onde podem realizar o descarte correto. A ausência de informações é um fator predominante para o desprezo indevido de medicamentos.

Desta forma, enquanto não for possível a criação de um gerenciamento de descarte de medicamentos gerados nas residências, é de extrema importância investir em orientação para a conscientização da população sobre o impacto do descarte incorreto destes.

\section{REFERÊNCIAS}

BILA, D. M.; DEZOTTI, M. Fármacos no meio ambiente. Quim. Nova, v. 26, n. 4, p. 523530, 2003.

BLANKENSTEIN, G. M.; PHILIPPI JUNIOR, A. O descarte de medicamentos e a política nacional de resíduos sólidos: uma motivação para a revisão das normas sanitárias. $R$. Dir. Sanit, São Paulo, v. 19, n. 1, p. 50-74, 2018.

BRASIL. Ministério da Saúde. Agência Nacional de Vigilância Sanitária (Anvisa). Resolução da Diretoria Colegiada - RDC № 222, de 28 de março de 2018. Dispõe sobre o Regulamento Técnico para o gerenciamento de resíduos de serviços de saúde.

BRASIL. Ministério do Meio Ambiente. Conselho Nacional do Meio Ambiente (Conama). Resolução $N^{\circ} 358$, de 29 de abril de 2005. Dispõe sobre o tratamento e a disposição final dos resíduos dos serviços de saúde e dá outras providências. Brasília, 2005.

FERREIRA, C. L. et al. Análise do conhecimento da população sobre descarte de medicamentos em Belo Horizonte - Minas Gerais. Interfaces Cientificas - Saúde e Ambiente, v. 3, n. 2, p. 9-18, 2015.

FERNANDES, R. M. et al. Armazenamento e descarte dos medicamentos vencidos em farmácias caseiras: problemas emergentes para a saúde pública. Einstein, São Paulo, v. 1, n. 18, p. 1-6, 2019.

JORGE JOÃO, W. S. J. Descarte de medicamentos. Pharmacia Brasileira, n. 82, 2011.

LENHARDT, H. E. et al. O descarte de medicamentos no Bairro Grande Terceiro, Cuiabá-MT. Cient. Ciênc. Biol. Saúde, v. 16, n. 1, p. 5-8, 2014.

LIMA, Y. N. H et al. Investigação sobre o descarte de medicamentos residenciais com acadêmicos do Centro Universitário Celso Lisboa - UCL. Revista Presença, v. 2, n. 5, p. 42-60, 2016.

MACIEL, H. J. et al. Estratégias de implantação do plano de descarte de medicamentos vencidos na cidade de Teófilo Otoni-MG. Revista Multidisciplinar do Nordeste Mineiro Unipac, p. 330-338, 2018.

Editora Unijuí - Revista Contexto \& Saúde - ISSN 2176-7114 - v. 21, n. 43, jul./set. 2021 
MAIA, M.; GIORDANO, F. Estudo da situação atual de conscientização da população de Santos a respeito do descarte de medicamentos. Revista Ceciliana, v. 4, n. 1, p. 24-28, 2012.

MEDEIROS, M. S. G.; MOREIRA, L. M. F.; LOPES, C. C. G. O. Descarte de medicamentos: programas de recolhimento e novos desafios. Rev. Ciênc. Farm. Básica Apl., v. 35, n. 4, p. 651-662, 2014.

PINTO, G. M. F et al. Estudo do descarte residencial de medicamentos vencidos na região de Paulínia (SP), Brasil. Eng. Sanit. Ambient., v. 19, n. 3, p. 219-224, 2015.

SOUZA, C. P. F. A.; FALQUETO, E. Descarte de medicamentos no meio ambiente no Brasil. Rev. Bras. Farm., v. 96, n. 2, p. 1.142-1.158, 2015.

TRIBESS JUNIOR, A.; BELLAVER, E. H.; ZANCANARO, V. Uso racional e descarte de medicamentos no grupo hiperdia no Bairro Nossa Senhora Salete no município de Caçador/SC. Saúde Meio Ambiente, v. 7, n. 1, p. 81-90, 2018.

UEDA, J. et al. Impacto ambiental do descarte de fármacos e estudo da conscientização da população a respeito do problema. Revista Ciências do Ambiente On-Line, v. 5, n. 1, 2009.

VAZ, V. K.; FREITAS, M. M.; CIRQUEIRA, Z. J. Investigação sobre a forma de descarte de medicamentos vencidos. Cenarium Pharmacêutico, v. 4, n. 4, 2011. 